\title{
Tratamiento quirúrgico de linfedema peneano secundario a hidrosadenitis supurativa
}

E. García-Tutor*, J. Botellé del Hierro*, A. San Martín Maya*, J. Castro García*, A. España**, J. Fernández Montero***, J.E. Robles García***

*Departamento de Cirugía Plástica, Estética y Reparadora. **Departamento de Dermatología. ***Departamento de Urología. Clínica Universitaria. Universidad de Navarra. Pamplona.

Resumen: El linfedema de pene y escroto es una enfermedad rara en los países desarrollados, aunque es relativamente frecuente en países orientales. La causa más frecuente es la filariasis ${ }^{1}$, si bien en nuestro medio suele encontrarse asociada a procesos neoplásicos, inflamatorios, postquirúrgicos, secuelas por radioterapia, disbalances hidroelectrolíticos y procesos idiopáticos.

Presentamos un paciente de 22 años que presenta linfedema penoescrotal secundario a hidrosadenitis inguinal de larga evolución. Tras fracasar el tratamiento conservador se somete a tratamiento quirúrgico consistente en resección de piel y tejido celular subcutáneo del pene hasta fascia de Buck y cobertura con injerto de piel parcial de muslo. Dadas las características de la zona anatómica, el tratamiento conservador es difícil y poco satisfactorio, por lo que la exéresis completa de la piel y la cobertura posterior con injertos es una opción terapéutica segura y eficaz.

Palabras clave: Linfedema. Penoescrotal. Hidrosadenitis. Elefantiasis. Injerto cutáneo. Pene. Tratamiento quirúrgico.

Abstract: Penoscrotal lymphedema is a rare disease in the developed countries, although it is relatively frequent in tropical countries. The most common cause is filariasis, although in our practice usually is associate to neoplasic and inflammatory processes, surgery, radiotherapy, hidroelectrolitic disbalances and idiopathic.

We present a 22 years old patient with penoscrotal lymphedema due to hidradenitis suppurativa. After unsuccessful medical treatment, was performed a total excision of the penile skin and subcutaneous tissue to Buck's fascia. Split thickness skin grafts were used to cover the defect. Even medical management of penoscrotal lymphedema is not effective for most patients, surgery is a safe and effective procedure that gives excellent functional and cosmetic results.

Keywords: Lymphedema. Penoscrotal. Hidradenitis. Elephantiasis. Skin graft. Penis. Surgical treatment.

Dr. E. García-Tutor

Departamento de Cirugía Plástica Reparadora y Estética

Clínica Universitaria. Universidad de Navarra 31008 Pamplona (Navarra)

e-mail: egtutor@unav.es 
El linfedema penoscrotal es una enfermedad rara en los países desarrollados ${ }^{1}$. Causa dolor, deformidad, dificultad para orinar, disminución de la potencia sexual y, en los casos extremos, dificultad para la bipedestación y la marcha. Todos los procesos que pueden causarla tienen en común que provocan una obstrucción en los vasos linfáticos regionales, observándose al microscopio una dilatación de los conductos linfáticos distales al proceso obstructivo.

Los procesos que pueden causarlo se agrupan en inflamatorios, postquirúrgicos, neoplásicos, secundarios a secuelas post-radioterapia, por disbalances hidroelectrolíticos y formas idiopáticas.

De entre todas las causas infecciosas, la más frecuente es la filariasis siendo ésta la causa global más frecuente a escala mundial. Se ha relacionado también aisladamente con otras enfermedades infecciosas como con la hidrosadenitis supurativa ${ }^{2}$ así como después de infecciones estreptocócicas ${ }^{3}$ o tuberculosas.

Las etiologías más frecuentes de origen neoplásico son el cáncer de próstata y las metástasis ganglionares inguinales. También las enfermedades granulomatosas como la enfermedad de Crohn, la artritis reumatoide y la sarcoidosis pueden ser causa de linfedema penoscrotal ${ }^{3}$. Otras situaciones como disbalances hidroelectrolíticos (como la hipoproteinemia) y de igual manera los procedimientos quirúrgicos, como las linfadenectomías inguinales, también pueden originarlo. Más raramente se ha descrito linfedema genital tras el uso de inyecciones de parafina o silicona en el pene o con el uso de bandas constrictivas alrededor del escroto ${ }^{4}$.

\section{CASO CLINICO}

Presentamos un paciente varón de 22 años afecto de linfedema de pene y escroto secundario a hidrosadenitis inguinal de cuatro años de evolución (Fig. 1). El cultivo del material purulento reveló la presencia de S. aureus y E. coli. Recibió tratamiento con amoxicilina-clavulánico, tetraciclinas, levofloxacino e isotretinoína éste último durante más de un año, mejorando las lesiones inguinales aunque no el linfedema.

Se realizó linfografía isotópica con sulfuro de renio marcado con 99mTc observándose retraso en la migración del radiotrazador e importante disminución del número de ganglios inguinales y de la cadena ganglionar iliaca, de forma bilateral. Mediante TAC abdominopélvico se descartó la presencia de procesos obstructivos en esta localización. Una biopsia de piel del escroto mostró la epidermis respetada, con dilatación de vasos linfáticos en dermis, de contorno irregular revestidos con endotelio maduro, sin alteraciones del estroma, con diagnóstico de linfangiectasia cutánea. Estos hallazgos junto a las manifestaciones clínicas del paciente, fueron característicos de linfedema peneano y escrotal secundario a hidradenitis supurativa inguinal.

A la vista de los resultados y de que la principal preocupación del paciente era el aumento en el volumen de su pene, se realizó mediante anestesia general y previo sondaje vesical, circuncisión y exéresis completa de piel y tejido celular subcutáneo hasta la fascia de Buck, respetando la piel del escroto (Fig. 2). Tras la infiltración de 10 
microgramos de alprostadil intracavernoso se realizó cobertura del cuerpo peneano con injerto cutáneo de espesor parcial de muslo, de forma espiroidea, sobre la fascia de Buck (Fig.3). Tras siete días levantamos la cura, habiendo prendido en su totalidad. El resultado al mes es satisfactorio, sin recidiva del edema ni incurvaciones en la erección (Fig. 4).

\section{DISCUSIÓN}

El linfedema penoscrotal es una entidad poco frecuente en nuestro medio, aunque común en países en los que la filariasis es endémica.

Como se describe en los trabajos de $\mathrm{McDougal}^{3}$, salvo en el caso poco común en que el linfedema sea autolimitado y no produzca un daño permanente en la piel, se precisa de un tratamiento quirúrgico agresivo, ya que en el linfedema crónico se producen cambios irreversibles en la piel y el tejido celular subcutáneo. Los objetivos que se persiguen con este tratamiento son los siguientes: un resultado estético aceptable, que posibilite una actividad sexual normal, que cree un ambiente adecuado para la espermatogénesis ${ }^{5} \mathrm{y}$ que minimice el riesgo de recurrencias ${ }^{3}$.

Las diferentes alternativas quirúrgicas tienen en común la eliminación completa de piel y tejido celular subcutáneo y su posterior cobertura con colgajos cutáneos o con injertos de espesor parcial ${ }^{3,4,6}$. En el caso del linfedema peneano la opción más aceptada es la escisión de piel y tejido celular subcutáneo hasta la fascia de Buck recubriendo posteriormente el defecto con injertos de piel de espesor parcial obtenidos de muslo o abdomen.

En el escroto existen tres alternativas. La primera sería la escisión completa de toda la piel y el tejido celular subcutáneo y su recubrimiento con un colgajo de la piel posterolateral del escroto que, en muchos casos, no se halla afectada por el linfedema ${ }^{1,3}$. Así se logra construir un neoscroto de características muy similares al anterior, permitiendo una adecuada espermatogénesis ${ }^{6}$. Otra opción sería el recubrimiento con colgajos locales de muslo o abdomen ${ }^{3}$, aunque en este caso sería discutible si el ambiente que se crea en el escroto sería favorable para la espermatogénesis.

Hay que tener en cuenta que, en estas dos últimas técnicas, la eliminación del riesgo de recurrencia es dudoso por lo que muchos autores prefieren recubrir el defecto con injertos de espesor parcial que no parecen interferir en el proceso de termoregulación de la espermatogénesis. Además proporciona un resultado estético aceptable eliminando, en principio, el riesgo de recurrencia al extirpar todo el tejido afectado.

Debido a la dificultad que presenta realizar un tratamiento conservador en la zona y tras consultar la literatura revisada, creemos que la exéresis completa de la piel y tejido celular subcutáneo y cobertura posterior es una buena opción terapéutica. 


\section{REFERENCIAS}

1. Dandapat MC, Mohapatro SK, Patro SK. Elephantiasis of the penis and scrotum. A review of 350 cases. American Journal of Surgery, 1985;149(5): 686-690.

2. Konety BR, et al. Scrotal elephantiasis associated with hidradenitis suppurativa. Plastic and Reconstructive Surgery 1996;97(6):1243-1245.

3. McDougal WS. Lymphedema of the external genitalia. The Journal of Urology 2003;170(3): 711-716.

4. Malloy TR, Wein AJ, Gross P. Scrotal and penile lymphede-ma: surgical considerations and management. The Journal of Urology 1983;130(2): 263-265.

5. Steinberg J, Kim ED, McVary KT. A surgical approach to penoscrotal lymphedema. The Journal of Urology 1996;156 (5):1770.

6. Ross JH, et al. Primary lymphedema of the genitalia in children and adolescents. The Journal of Urology 1998;160(4): 1485-1489. 


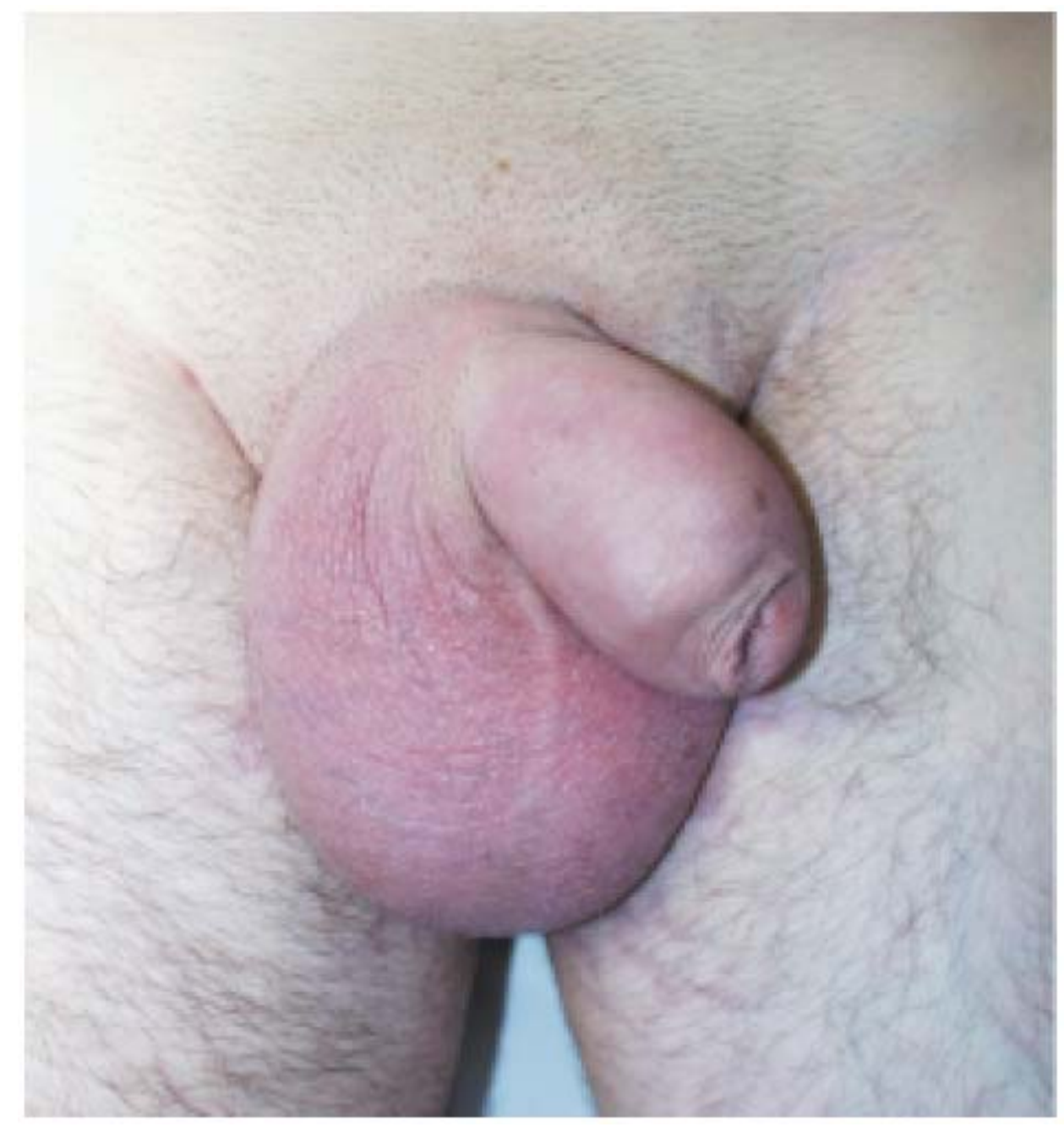

Figura 1. Situación preoperatoria 


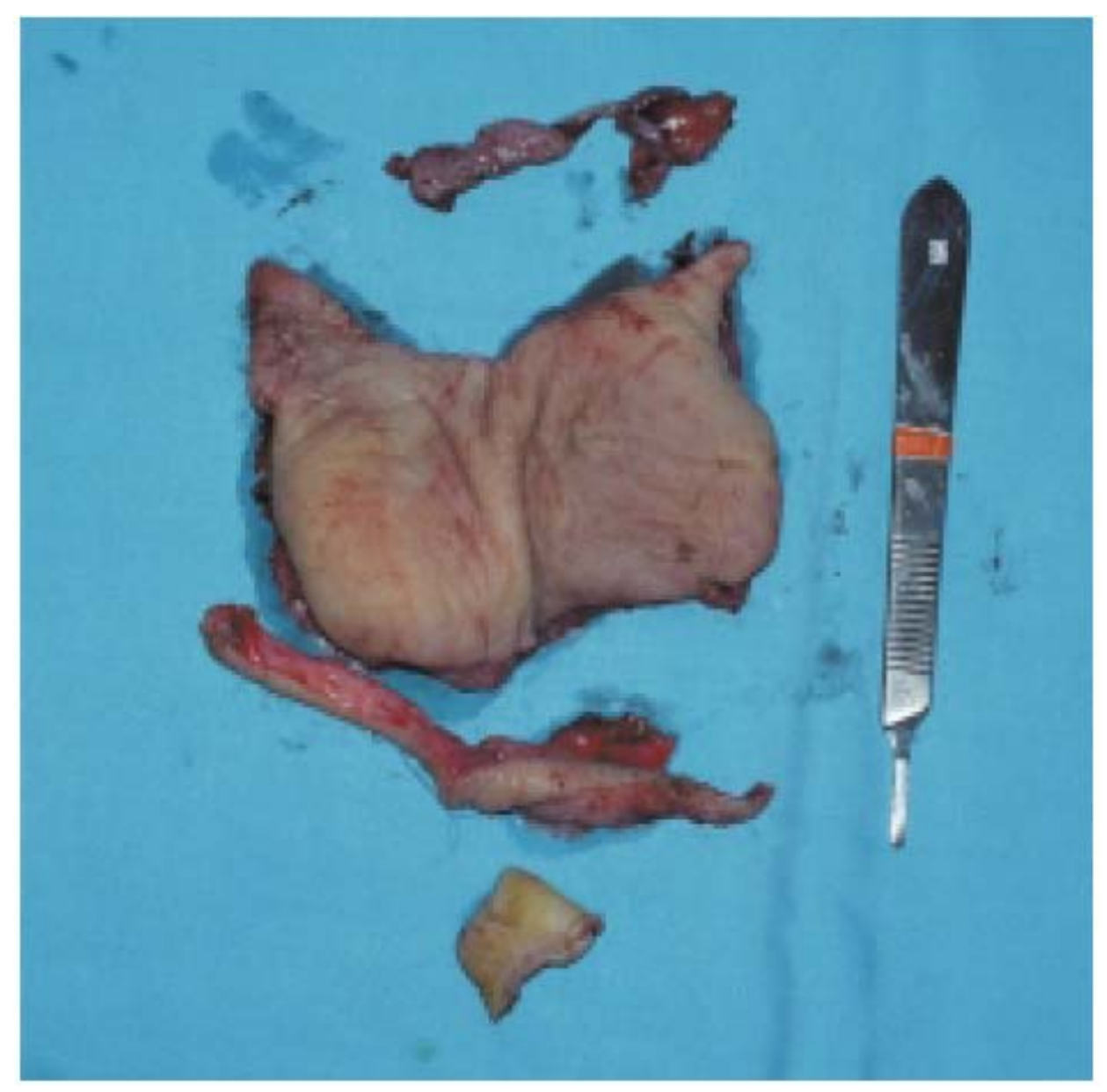

Figura 2. Pieza quirúrgica 


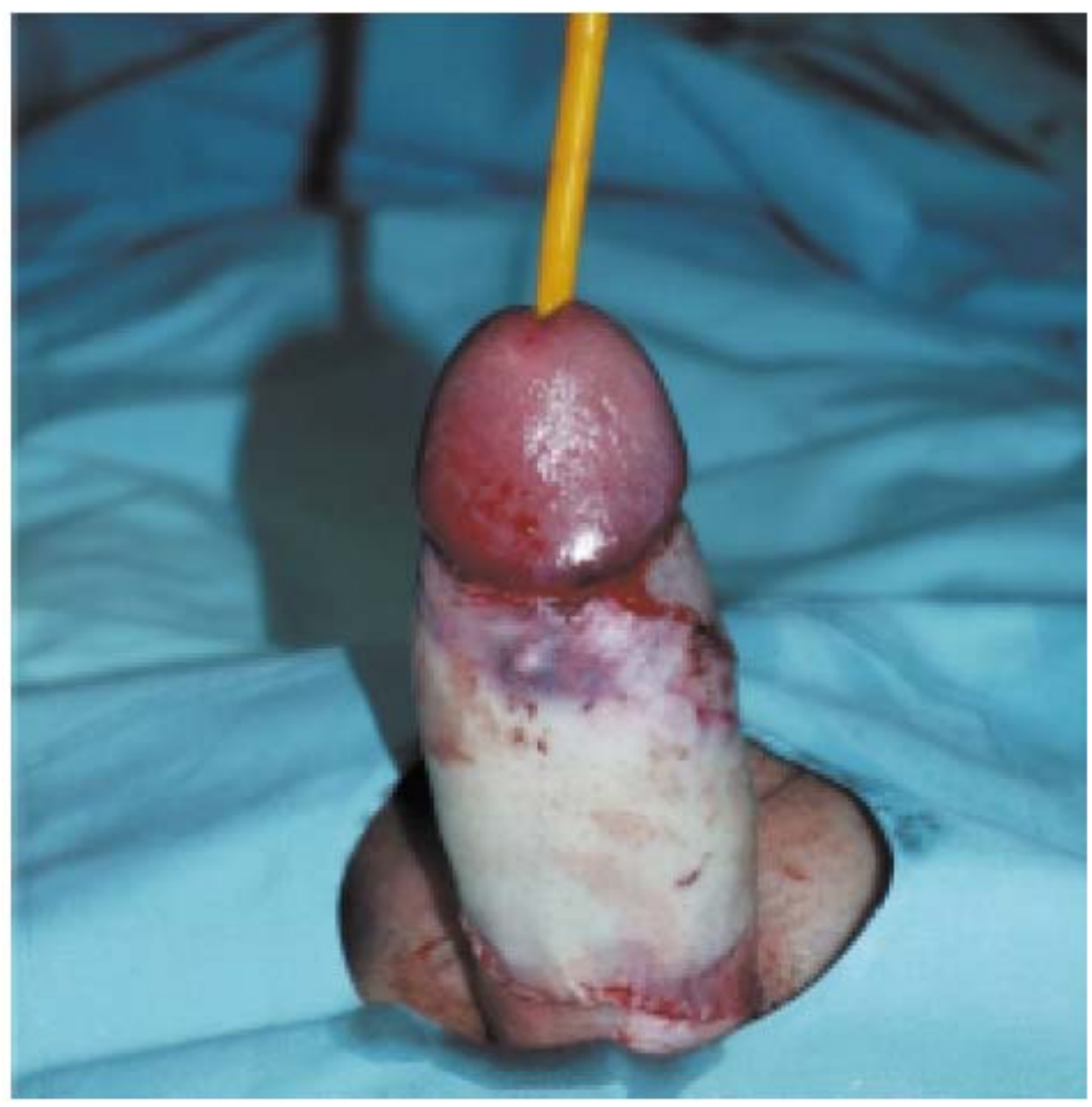

Figura 3. Imagen quirúrgica del pene injertado 


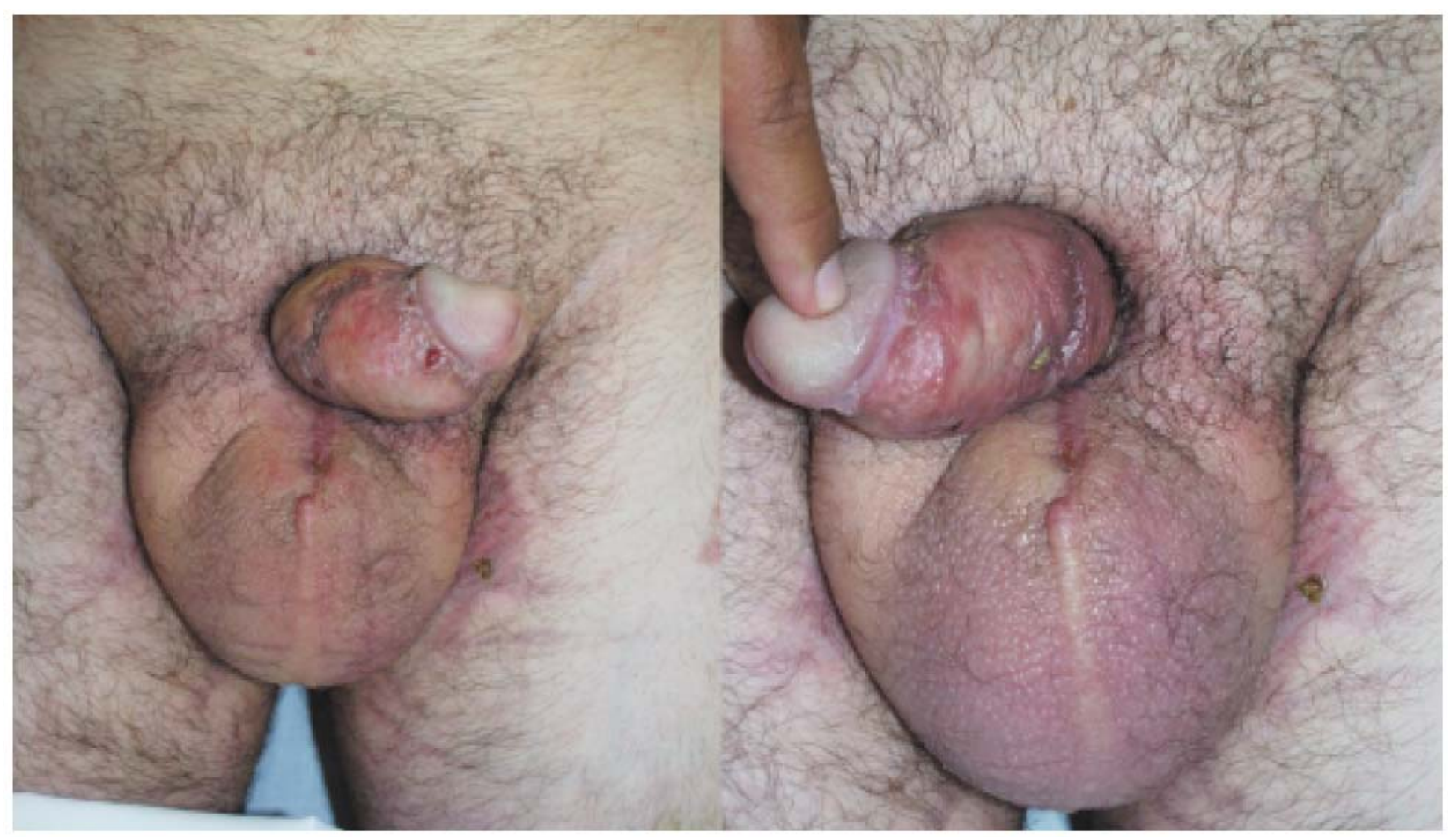

Figura 4. Situación postoperatoria al mes de la intervención 